\title{
Tensor RG calculations and quantum simulations near criticality
}

\author{
Y. Meurice ${ }^{\star a}$, A. Bazavov ${ }^{a, b, d}$, Shan-Wen Tsai ${ }^{a}$, J. Unmuth-Yockey ${ }^{b}$, Li-Ping Yang ${ }^{c}$, \\ Jin Zhang ${ }^{a}$ \\ ${ }^{a}$ Department of Physics and Astronomy, University of Iowa, Iowa City, IA 52242, USA \\ ${ }^{b}$ Department of Physics and Astronomy, University of California, Riverside, CA 92521, USA \\ ${ }^{c}$ Department of Physics, Chongqing University, Chongqing 401331, China \\ ${ }^{d}$ Department of Physics and Astronomy, Michigan State University, East Lansing, Michigan \\ 48824, USA \\ E-mail: yannick-meurice@uiowa.edu
}

\begin{abstract}
We discuss the reformulation of the $\mathrm{O}(2)$ model with a chemical potential and the Abelian Higgs model on a 1+1 dimensional space-time lattice using the Tensor Renormalization Group (TRG) method. The TRG allows exact blocking and connects smoothly the classical Lagrangian approach to the quantum Hamiltonian approach. We calculate the entanglement entropy in the superfluid phase of the $\mathrm{O}(2)$ model and show that it approximately obeys the logarithmic CalabreseCardy scaling obtained from Conformal Field Theory (CFT). We calculate the Polyakov loop in the Abelian Higgs model and discuss the possibility of a deconfinement transition at finite volume. We propose Bose-Hubbard Hamiltonians implementable on optical lattices as quantum simulators for CFT models.
\end{abstract}

34th annual International Symposium on Lattice Field Theory

24-30 July 2016

University of Southampton, UK

\footnotetext{
*Speaker.
} 


\section{Introduction}

Recent calculation using the Tensor Renormalization Group (TRG) method [1, 2, 3, 4, 5, 6, $7,8]$ have shown that blocking methods can complement sampling methods for models studied in lattice field theory. The ultimate goal of the method is to approach the continuum limit of lattice models in terms of fixed points of the TRG transformation [2,3]. A first step in this direction is to control the finite dimensional approximations.

The $O(2)$ model with a chemical potential in $1+1$ dimensions has a complex action and a sign problem if simulated with MC methods, but can be handled with a worm algorithm [9] and the TRG method $[5,10]$. For sufficiently large $\beta$ or $\mu$, the model has a superfluid phase where we expect to have a conformal field theory (CFT) with central charge $c=1$. This idea can be tested by calculating the von Neumann and Rényi entanglement entropy (EE) and comparing their finite size scaling with the CFT Calabrese-Cardy scaling [11] which predicts a logarithmic growth with the size of the system and a coefficient which is the central charge divided by an integer depending on the type of entropy and the boundary conditions. This scaling appears very clearly for the von Neumann EE with periodic boundary conditions, but it was realized after the conference that for the second order Rényi entropy, $S_{2}$, with open boundary conditions, large subleading corrections appear and need to be taken into account. Fortunately, they can also be understood in the context of CFT [12] and allowed us to check the validity of the expectation that $c=1[13,14]$.

By gauging the $O(2)$ model, we obtain the Abelian Higgs model. By keeping the gauge coupling small enough when $N_{s}^{2}$ increases, with $N_{s}$ the spatial number of sites, we can use the gauge field to probe the $O(2)$ model. Below, we discuss the data collapse for the Polyakov loop calculated in the scaling limit where $g^{2}$ decreases like $1 / N_{s}^{2}$. We show that this observable singles out the transition to topological order more sharply than $S_{2}$.

Recently, it has been possible to measure $S_{2}$ in quantum simulations for the Bose-Hubbard model using optical lattices [15] with a beamsplitter operation proposed in Ref. [16]. This experimental setup can quantum simulate the $O(2)$ model with a chemical potential [10] using only one species of bosonic atom provided that the chemical potential is large enough. After the conference, we developed more specific experimental methods to measure the central charge using existing optical lattice experiments [13]. This opens the possibility of exploring CFT using one-dimensional optical lattices.

\section{The Tensor Renormalization Group (TRG) method}

The TRG method provides exact blocking formulas for spin and gauge models [4]. The blocking separates the degrees of freedom inside the block (integrated over), from those kept to communicate with the neighboring blocks. The only approximation is the truncation in the number of "states" kept. It applies to many lattice models: the Ising model [1, 2, 3], the $O(2)$ model [4, 17], the $O(3)$ model [4, 18], the $S U(2)$ principal chiral model, Abelian and $S U(2)$ gauge theories [4], the Schwinger model [6, 19], Gross-Neveu model [8], and $\mathrm{CP}(\mathrm{N}-1)$ models [7]. More recent progress has been reviewed in the plenary talk by Shinji Takeda [20].

The TRG method relies on algebraic manipulations of positive matrices and seems free of sign problems. It can handle problems with complex temperature [5] and real chemical potential [10] . 
It has been checked [21] with a worm algorithm [9]. It has been used to study fixed points [2, 3], however the effects of truncations are not well-understood in this context. The TRG approach connects easily to the Hamiltonian picture and provides spectra which can be used for real time calculations. It has been used to design quantum simulators for the $O(2)$ model [10] and the Abelian Higgs model [22].

\section{The Rényi entanglement entropy}

The TRG can be used to calculate various types of entanglement entropy (EE). We consider the subdivision of a time slice $A B$ into two parts $A$ and $B$ (two halves in our calculation). After tracing over $B$, we obtain the reduced density matrix $\hat{\rho}_{A} \equiv \operatorname{Tr}_{B} \hat{\rho}_{A B}$ and we can calculate the Von Neumann entropy

$$
S_{\text {vonNeumann }}=-\sum_{i} \rho_{A_{i}} \ln \left(\rho_{A_{i}}\right),
$$

where $\rho_{A_{i}}$ are the eigenvalues of $\hat{\rho}_{A}$. We then use the TRG blocking method until $A$ and $B$ are each reduced to a single site. With this method, we can only calculate the entanglement entropy for regions that contain a number of sites that is a power of 2 . We can also compute the $n$-th order Rényi EE defined as:

$$
S_{n}(A) \equiv \frac{1}{1-n} \ln \left(\operatorname{Tr}\left(\hat{\rho}_{A}^{n}\right)\right) .
$$

In the limit where $n \rightarrow 1^{+}$, we recover the von Neumann EE. Numerical results for the $O(2)$ model in $1+1$ dimensions with a chemical potential are shown in Fig. 1 for open (OBC) and periodic boundary conditions (PBC) in the Kosterlitz-Thouless (KT) phase. The approximately linear be-


Figure 1: The Von Neumann and second order Renyi EE for OBC (left) and PBC (right).

havior in $\ln \left(N_{s}\right)$ is consistent with the Calabrese-Cardy scaling which predicts

$$
S_{n}\left(N_{s}\right)= \begin{cases}K_{n}+\frac{c(n+1)}{6 n} \ln \left(N_{s}\right) & \text { for PBC } \\ K_{n}^{\prime}+\frac{c(n+1)}{12 n} \ln \left(N_{s}\right) & \text { for OBC. }\end{cases}
$$

The constants $K$ are non-universal and different in the four situations considered ( $n=1,2$ with PBC and $\mathrm{OBC}$ ). $S_{2}$ can also be used to approximately localize the superfluid phase (where the particle 


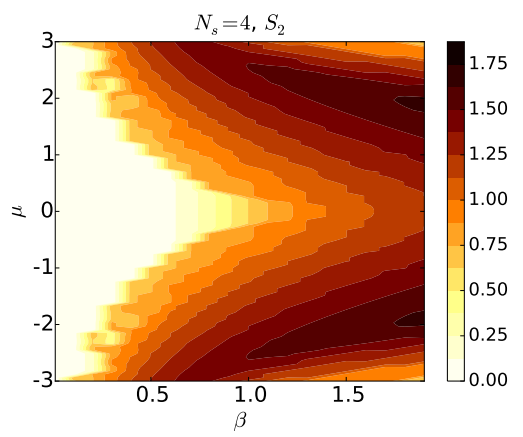

Figure 2: The second order Rényi $\mathrm{EE}\left(S_{2}\right)$ with $N_{s}=4$ and $\mathrm{OBC}$ in the $\mu-\beta$ plane.

density increases with the chemical potential). This is illustrated in Fig. 2. It shares some features with the $O(3)$ model with a chemical potential [23] which can also be quantum simulated [24].

In order to check with the Density Matrix Renormalization Group (DMRG) method, the time continuum limit can be achieved by increasing $\beta_{\tau}$ while keeping constant the products $\beta_{s} \beta_{\tau}=2 \tilde{J} / \tilde{U}$ and $\mu \beta_{\tau}=\tilde{\mu} / \tilde{U}$. This defines the rotor Hamiltonian:

$$
\hat{H}=\frac{\tilde{U}}{2} \sum_{x} \hat{L}_{x}^{2}-\tilde{\mu} \sum_{x} \hat{L}_{x}-2 \tilde{J} \sum_{\langle x y\rangle} \cos \left(\hat{\theta}_{x}-\hat{\theta}_{y}\right)
$$

with $\left[\hat{L}_{x}, \mathrm{e}^{i \hat{\theta}_{y}}\right]=\delta_{x y} \mathrm{e}^{i \hat{\theta}_{y}}$. For quantum simulation purposes, these commutation relations can be approximated for finite integer spin [10]. In the following we focus on the spin-1 approximation which can also be implemented in the classical system by setting the tensor elements to zero for space and time indices strictly larger than 1 in absolute value. The correspondence between the two methods can be checked with a Density Matrix Renormalization Group (DMRG) method which optimizes the EE and allows one to calculate observables for any number of sites. When applied to $S_{2}$ with OBC, large subleading corrections become apparent $[13,14]$ for the intermediate values of $L$ not shown in Fig. 2.

\section{The Polyakov loop in the Abelian Higgs model}

The TRG method was used to calculate the partition function of the Abelian Higgs model [22]. We attach a $B^{(\square)}$ tensor to every plaquette, a $A^{(s)}$ tensor to the spatial links (they cross these links and point in the Euclidean time direction) and a $A^{(\tau)}$ tensor to the temporal links (they point in the spatial direction, see figures in [22]). The partition function can be written as

$$
\begin{aligned}
& Z=\left(I_{0}\left(\beta_{p l}\right) I_{0}\left(2 \kappa_{s}\right) I_{0}\left(2 \kappa_{\tau}\right)\right)^{V} \times \\
& \operatorname{Tr}\left[\prod_{h, v, \square} A_{m_{u p}}^{(s)} m_{\text {down }} A_{m_{\text {right }} m_{\text {left }}}^{(\tau)} B_{m_{1} m_{2} m_{3} m_{4}}^{(\square)}\right] .
\end{aligned}
$$

A few remarks are in order. The quantum numbers on the links are completely determined by the quantum numbers on the plaquettes. The plaquette quantum numbers are the dual variables. The reformulation is manifestly gauge invariant. If we impose periodic boundary conditions on the 
plaquettes, we can only have neutral states (Gauss law). For related questions in QED, see Ref. [25]. It is however possible to probe the charge sectors by introducing Polyakov loops.

We consider Polyakov loops wrapping around the Euclidean time direction: $\left\langle P_{i}\right\rangle=\left\langle\prod_{j} U_{(i, j), \tau}\right\rangle$. With spatial periodic boundary conditions, the insertion of the Polyakov loop shown in red in Fig. 3 forces the presence of a scalar current (green) in the opposite direction (left) or it may be compensated by another Polyakov loop in the opposite direction (right).


Figure 3: Polyakov loop (red), current (green) in the opposite direction (left); compensation by another Polyakov loop (right).

Some interesting data collapse has been found for the Polyakov loop $P$. The relation with the mass gap $\Delta E$ is that $-\ln (P) \simeq C+N_{\tau}(\Delta E)$. We then use the fact that in absence of gauge coupling, the gap in the KT phase decreases like $1 / N_{s}$, while the gauge fields create horizontal lines of 1 's in Fig. 3 and so $\Delta E \simeq A / N_{s}+B g^{2} N_{s}+\ldots$ and we obtain a data collapse for $N_{s} \Delta E=F\left(g^{2} N_{s}^{2}\right)$. Numerical calculations shown in Fig. 4 give support to this idea.


Figure 4: (left) The Polyakov loop as function of $\kappa=\beta_{s} / 2$, the increase in sharpness with volume makes it look like an order parameter; (right) data collapse for $N_{s} \Delta E$.

\section{Quantum Simulators}

There has been a recent interest in using cold atoms trapped in optical lattices for quantum simulating spin and gauge models studied by lattice gauge theorists [26]. This means using the interaction of polarizable cold atoms trapped in a periodic potential and their tunneling properties to built a system evolving at real time with a Hamiltonian similar to one of the models considered. 
There are no sign problems and real time evolution occurs at physical time. So far linear sizes reached in experiment can be of order 100-200 and are expected to reach 1000 soon. Our approach is based on the TRG formulation of lattice gauge theory and is manifestly gauge invariant.

So far, the remarkable theory/experiment reached for the Bose-Hubbard model is just a source of inspiration in the context of lattice gauge theory and a proof of principle is needed. We hope that Ref. [13] is a step in this direction. In this recent preprint, we compared the second-order Rényi EE, $S_{2}$, of the classical $O(2)$ model with a chemical potential on a $1+1$ dimensional lattice, and a quantum Bose-Hubbard Hamiltonian that can be simulated with cold atoms on a one-dimensional optical lattice. Both models have a superfluid phase where we expect $S_{2}$ to follow the CalabreseCardy scaling. Near half-filling and for a small hopping parameter $J, S_{2}$ is almost identical for both models. We proposed to amend the existing experimental setup to measure $S_{2}$ by adiabatically reaching the ground state of twin tubes half-filled with ${ }^{87} \mathrm{Rb}$ atoms with small $J$; this is in contrast to existing experiments with density one at larger $J$.

\section{Conclusions}

The TRG formulation allows reliable calculations of the phase diagram and spectrum of the $1+1$ dimensional $O(2)$ model with a chemical potential. Calculations of the von Neumann and Rényi EE for the $O(2)$ model in the superfluid phase at increasing $N_{s}$ seem consistent with CFT of central charge 1. We have proposed a gauge-invariant approach for the quantum simulation of the abelian Higgs model. Calculations of the Polyakov loop at finite $N_{s}$ and small gauge coupling shows interesting behavior. We obtained a nice data collapse at weak gauge coupling.

Acknowledgments. This research was supported in part by the Department of Energy under Award Numbers DOE grant DE-FG02-05ER41368, DE-SC0010114 and DE-FG02-91ER40664, the NSF under grant DMR-1411345 and by the Army Research Office of the Department of Defense under Award Number W911NF-13-1-0119. L.-P. Yang was supported by Natural Science Foundation for young scientists of China (Grants No.11304404) and Research Fund for the Central Universities(No. CQDXWL-2012-Z005). Parts of the numerical calculations were done at the Argonne Leadership Computational Facilities.

\section{References}

[1] Z. Y. Xie, J. Chen, M. P. Qin, J. W. Zhu, L. P. Yang, and T. Xiang, Phys. Rev. B 86 (Jul, 2012) 045139, [arXiv:1201.1144].

[2] Y. Meurice, Phys. Rev. B 87 (2013), 064422, [arXiv: 1211.3675$].$

[3] E. Efrati, Z. Wang, A. Kolan, and L. P. Kadanoff, Rev. Mod. Phys. 86 (2014) 647, [arXiv:1301.6323].

[4] Y. Liu, Y. Meurice, M. P. Qin, J. Unmuth-Yockey, T. Xiang, Z. Y. Xie, J. F. Yu, and H. Zou, Phys. Rev. D 88 (2013) 056005, [arXiv: 1307.6543 ].

[5] A. Denbleyker, Y. Liu, Y. Meurice, M. P. Qin, T. Xiang, Z. Y. Xie, J. F. Yu, and H. Zou, Phys. Rev. D 89 (2014), 016008, [arXiv:1309.6623].

[6] Y. Shimizu and Y. Kuramashi, Phys. Rev. D 90 (2014), 014508, [arXiv: 1403.0642 ]. 
[7] S. Takeda and Y. Yoshimura, PTEP 2015 (2015), 043B01, [arXiv: 1412 . 7855].

[8] H. Kawauchi and S. Takeda, arXiv:1511.00348; H. Kawauchi and S. Takeda, Phys. Rev., vol. D93, no. 11, p. 114503, 2016.

[9] D. Banerjee and S. Chandrasekharan, Phys.Rev. D 81 (2010) 125007, [arXiv: 1001.3648 ].

[10] H. Zou, Y. Liu, C.-Y. Lai, J. Unmuth-Yockey, L.-P. Yang, A. Bazavov, Z. Y. Xie, T. Xiang, S. Chandrasekharan, S.-W. Tsai, and Y. Meurice, Phys. Rev. A 90 (2014) 063603 , [arXiv: 1403.5238$]$.

[11] P. Calabrese and J. L. Cardy, J.Stat.Mech. 0406 (2004) P06002, [hep-th / 0405152 ].

[12] P. Calabrese, M. Campostrini, F. Essler, and B. Nienhuis, Phys. Rev. Lett., vol. 104, p. 095701, Mar 2010; J. Cardy and P. Calabrese, Journal of Statistical Mechanics: Theory and Experiment, vol. 2010, no. 04, p. P04023, 2010.

[13] J. Unmuth-Yockey, J. Zhang, P. M. Preiss, L.-P. Yang, S.-W. Tsai, and Y. Meurice, arxiv-1611.05016.

[14] J. Unmuth-Yockey, J. Zhang, L.-P. Yang, A. Bazavov, S.-W. Tsai, and Y. Meurice, preprint in progress.

[15] R. Islam, R. Ma, P. M. Preiss, M. E. Tai, A. Lukin, M. Rispoli, and M. Greiner, Nature, vol. 528, p. 77, 2015; A. M. Kaufman, M. E. Tai, A. Lukin, M. Rispoli, R. Schittko, P. M. Preiss, and M. Greiner, Science, vol. 353, no. 6301, pp. 794-800, 2016.

[16] A. J. Daley, H. Pichler, J. Schachenmayer, and P. Zoller, Phys. Rev. Lett., vol. 109, p. 020505, Jul 2012.

[17] Y. Meurice, Y. Liu, J. Unmuth-Yockey, L.-P. Yang, and H. Zou, Sampling versus Blocking, PoS LATTICE2014 (2014) 319, [arXiv:1411.3392].

[18] J. F. Unmuth-Yockey, Y. Meurice, J. Osborn, and H. Zou, Tensor renormalization group study of the 2d O(3) model, PoS LATTICE2014 (2014) 325, [arXiv : 1411 . 4213].

[19] H. Saito, M. C. Bañuls, K. Cichy, J. I. Cirac, and K. Jansen, PoS LATTICE2014 (2014) 302, [arXiv:1412.0596].

[20] Shinji Takeda, these proceedings.

[21] L.-P. Yang, Y. Liu, H. Zou, Z. Y. Xie, and Y. Meurice, Phys. Rev. E, vol. 93, p. 012138, Jan 2016.

[22] A. Bazavov, Y. Meurice, S.-W. Tsai, J. Unmuth-Yockey, and J. Zhang, Phys. Rev. D, vol. 92, p. 076003 , Oct 2015.

[23] F. Bruckmann, C. Gattringer, T. Kloiber, and T. Sulejmanpasic, 2016, [arXiv:1607.02457] and these proceedings.

[24] C. Laflamme, W. Evans, M. Dalmonte, U. Gerber, H. Mejadaz, W. Bietenholz, U. J. Wiese, and P. Zoller, Annals Phys., vol. 370, pp. 117-127, 2016.

[25] Biagio Lucini, Agostino Patella, Alberto Ramos, and Nazario Tantalo, arXiv:1509.01636, JHEP02(2016)076.

[26] For recent reviews see: L. Tagliacozzo, A. Celi, A. Zamora, and M. Lewenstein, Annals Phys., vol. 330, pp. 160-191, 2013; U.-J. Wiese, Annalen Phys., vol. 525, pp. 777-796, 2013; E. Zohar, J. I. Cirac, and B. Reznik, Rept. Prog. Phys., vol. 79, no. 1, p. 014401, 2016. 